\title{
PAYS-BAS
}

\section{LE DOCTEUR M.-V.-C. GORI}

La Croix-Houge néerlandaise vient de perdre un de ses plus anciens et fidèles amis, en la personne de M. le docteur Morinus Willem Clémente Gori, lecteur à l'universilé d'Amsterdam et directeur du Comité de la Croix-Ronge de cette ville. Né le 7 septembre 1834, il est décédé à Hilversum (Hollande) le 26 décernbre 1890. C'était un infatigable travailleur, surtout quand il s'agissait d'améliorer le sort des soldats et des blessés. Médecin militaire de 1856 à 1870, il put juger par lui-même des imperfections du service sanitaire dans l'armée. Aussi s'attacha-t-il avec ardeur à la CroixRouge, et, même après la guerre franco-allemande, lorsqu'il eut quilté le service pour pratique: civilement comme oculiste, la chirurgie militaire resta son étude de prédilection. Il fut délégué à plusieurs expositions, par exemple à celles de Washington, de Vienne, de Londres, où il siégea dans les jurys et au sujet desquelles il publia de savants rapports, dont la Croix-Ronge a retiré un grand profit. La perte d'un collaborateur aussi précieux a été donloureusement ressentie par la Croix-Ronge néerlandaise, aux regrets de laquelle nous nous associons.

Nous avons sous les yeux la liste des publications du docteur Gori, qui toutes se rapportent à la chirurgie militaire et quelquesunes plus spécialement à la Croix-Rouge. Quinze d'entre elles sont écrites en hollandais, mais quatre, dont nous reproduisons les titres, ont paru en français. Ce sont les snivantes :

Des hpitaux, tentes t baraques, essai sur l'hygiène hospitalière, 1872.

La chirurgie militaire et les Sociétés de secours à l'Exposition de Vienne, 1873.

Sur le transport des soldats malades et blessés en temps de guerre. International Medical Congress, 1881.

Sur le transport des malades el blessés par les voies ferrées dans los climats tropicaux. Congrès international de médecine des colonies, 1883. 